$1.35,1.72)$ were more likely, and those who were underweight $(0.42 ; 0.28,0.64)$ less likely, to share a household with an overweight or obese younger child. No difference was identified in relation to the reference child's sex (1.03; $0.95,1.12)$.

Conclusion Our findings suggest that, over a five-year period in a geographically-defined and ethnically-diverse population with high childhood obesity prevalence, linked NCMP and GP records can be used to identify households with a high burden of childhood obesity. Further analyses of household social, environmental and health characteristics is underway to identify potentially modifiable factors at the household level.

\section{OP57 INEQUALITIES IN BMI IN SCOTLAND, 2008-2018: A REPEAT CROSS-SECTIONAL STUDY}

KA Levin*. Public Health Directorate, NHS Greater Glasgow and Clyde, Glasgow, UK

\subsection{6/jech-2020-SSMabstracts.56}

Background Obesity is associated with diseases such as cardiovascular disease and diabetes as well as mental health. Previously it was shown that the socioeconomic inequalities in adult BMI stabilised in Scotland between 2008 and 2014. The aim of this study is to describe the change in socioeconomic inequalities in BMI between 2008 and 2018.

Methods Data from the annual cross-sectional Scottish Health Survey between 2008 and 2018 for those aged 1664 years were analysed. Survey years were grouped into 2008-2011, 2012-2015, 2016-2018 and the data were modelled using logistic modelling for outcome 'obese/less than obese' and linear modelling for outcome BMI, for males and females, adjusting for 10 year age group, survey year groupings and SIMD quintile. An interaction terms between year and SIMD and year and age were included. Slope Index of Inequality (SII) and Relative Index of Inequality (RII) were calculated. Data were also analysed by 10 -year age group.

Results Average BMI and prevalence of obesity increased from 27.2 and $24.9 \%$ in 2008 to 27.5 and $25.9 \%$ in 2018 among males, and from 27.3 and $26.5 \%$ in 2008 to 27.6 and $29.0 \%$ in 2018 among females. When broken down by age group, the majority of this increase is observed among females aged 25-34 years and 45-54 years. When the data were modelled, socioeconomic inequalities in BMI were observed for both males (eg average BMI was 0.48 (0.23, 0.73 ) more in SIMD1, most deprived, compared with SIMD 5 ) and females (eg OR of being obese in SIMD1 was 1.45 $(1.28,1.63)$ of SIMD5). There was a significant rise in BMI and obesity prevalence over time. An interaction term between survey year and SIMD, however showed that this rise was not observed in the two most affluent SIMD quintiles and the increase for the three less affluent SIMD quintiles rose in an approximately stepwise fashion, suggesting a widening of inequalities, eg for outcome obese/not obese, adjusting for age, sex, year and SIMD, an interaction term between SIMD and survey year showed an OR of obesity in 2008 in SMID1 of $1.62(1.42,1.85)$ that of SIMD5, but for each additional year, OR rose by $1.03(1.003,1.05)$ for SIMD1 relative to SIMD5. Both RII and SII saw a large increase between 2012-15 and 2016-18 for males and females.
Conclusion Socioeconomic inequalities in BMI have previously thought to have plateaued, however this study shows that inequalities are now increasing.

\section{OP58 AN INVESTIGATION INTO THE ASSOCIATIONS BETWEEN SOCIO-DEMOGRAPHIC FACTORS, FOOD SOURCES AND DIETARY QUALITY IN SMALL ISLAND DEVELOPING STATES}

${ }^{1} E$ Haynes, ${ }^{2} \mathrm{D}$ Bhagtani, ${ }^{1} \mathrm{C}$ Guell, ${ }^{3} \mathrm{IR}$ Hambleton, ${ }^{4} \mathrm{~V}$ lese, ${ }^{4} \mathrm{~J}$ Fesaitu, ${ }^{5} \mathrm{SE}$ Benjamin-Neelon, ${ }^{2} \mathrm{NG}$ Forouhi, ${ }^{1,2} \mathrm{~N}$ Unwin*. ${ }^{1}$ European Centre for Environment and Human Health, University of Exeter Medical School, Truro, UK; ${ }^{2}$ MRC Epidemiology Unit, University of Cambridge, Cambridge, UK; ${ }^{3}$ George Alleyne Chronic Disease Research Centre, University of the West Indies, Bridgetown, Barbados; ${ }^{4}$ Pacific Centre for Environment and Sustainable Development, University of the South Pacific, Suva, Fiji; ${ }^{5}$ Department of Health, Behavior and Society, Johns Hopkins Bloomberg School of Public Health, Baltimore, Maryland, USA

\subsection{6/jech-2020-SSMabstracts.57}

Background Globally, some of the highest rates of obesity and non-communicable diseases (NCDs) are found in Small Island Developing States (SIDS). Relatedly, there has been a decrease in consumption of local foods and an increasing reliance on imported foods that are generally energy dense and highly processed. This study aimed to apply a novel quantitative toolkit to investigate the relationship between dietary diversity (DD) and food source, food insecurity and NCD risk in two SIDS: Fiji and Saint Vincent and the Grenadines (VCT).

Methods A dietary toolkit was developed to collect individual-level information on type and frequency of food consumed, food sources, food insecurity and relevant socioeconomic and health data. Regional investigators and partners ensured context-relevant content and implementation. In Fiji and VCT respectively, 95 and 86 households were recruited. All adults and adolescents (15 years and above) living in households, sampled to provide exposure to urban, rural, higher and lower income areas, were surveyed ( $\mathrm{n}$ individuals=186 SVG; n=147 Fiji). Descriptive statistics and multiple linear regression, with DD as the dependant variable, adjusted for household sampling, were applied to explore associations between sociodemographic factors, food sources and dietary quality.

Results Mean DD score, of a possible score of 10 , was 3.7 (SD1.4) in Fiji and 3.8 (SD1.5) in VCT, and this was consistent across sex, age and body mass index. In both settings, more people sourced food by purchasing than any other means (Fiji $n=155(83 \%)$; VCT $n=136(93 \%)$ ). Regular consumption of own produce and regular food borrowing were associated with greater fruit consumption (difference in median number of servings/week: Fiji 1(95\%CI 0,2); VCT 5 (95\%CI 1,9) and Fiji 2(0,4); VCT 9(5,13)), respectively. Purchasing from a small shop was associated with higher consumption of sugar-sweetened beverages (Fiji 4(1,7); VCT 7 $(1,13))$. Multivariable analysis results, presented as adjusted regression coefficients $(\beta(95 \% \mathrm{CI}))$, indicated that purchasing from a small shop was inversely associated with DD (-0.52 ($0.91,-0.12) ; \mathrm{p}=0.011)$, as was rural residence $(-0.46(-0.92$, $0.00) ; \mathrm{p}=0.049)$. Borrowing food was positively associated with DD $(0.73(0.21,1.25) ; \mathrm{p}=0.006)$, as was age $(0.01$ $(0.00,0.03) ; \mathrm{p}=0.063)$ and higher education $(0.44(0.06$, $0.82) ; \mathrm{p}=0.023)$.

Conclusion Our findings suggest barriers and facilitators to diet quality and links with food sources in SIDS that may 
contribute to enhancing understanding of local food consumption and health. The findings indicate important avenues for further research, such as the role that food borrowing may play in ensuring dietary diversity in these regions.

On behalf of the CFaH Team.

\section{Thursday 10 September}

\section{Health Policy I}

\section{OP59 STAKEHOLDER NARRATIVES OF 'PROBLEMS' AND 'SOLUTIONS': ANALYSING THE 2018 HEALTH AND SOCIAL CARE COMMITTEE ANTIMICROBIAL RESISTANCE SUBMISSIONS IN THE UNITED KINGDOM}

${ }^{1}$ RE Glover*, 'NB Mays, ${ }^{1} \mathrm{MP}$ Petticrew, ${ }^{2} \mathrm{C}$ Thompson. ${ }^{1}$ Faculty of Public Health Policy, LSHTM, London, UK; ${ }^{2}$ Health and Social Care, University of Hertfordshire, Hatfield, UK

\subsection{6/jech-2020-SSMabstracts.58}

Background Antimicrobial resistance (AMR) is an area of global policy attention. Antibiotic resistance is often characterised as a 'wicked problem', because it (i) affects, and requires simultaneous action by, public, private, and third sector stakeholders, (ii) requires local, regional, national, and supranational buy-in (and implementation of strategic change) across low, middle, and high-income countries, and (iii) spans human, animal, and environmental health. The corollary to AMR being described as a wicked problem is that 'crisis' narratives have been adopted by public health policymakers and practitioners to marshal resources, attention, and public engagement. This AMR narrative has been co-opted at times, in order to privilege solutions promoted by and involving the private sector; with the co-optation of these solutions comes the risk of sequestering public sector funds to subsidise private sector work - in particular, in the pharmaceutical and medical diagnostics industries.

Methods There were 72 written submissions made to the 2018 'Antimicrobial resistance' House of Commons Health and Social Care Committee. The sectors represented in these submissions were industry, trade associations, non-governmental organisations, professional associations, academia, government, public private partnerships, and homeopathy proponents. We accessed these documents and extracted relevant data according to the theoretically-informed critical discourse analysis (CDA) framework that we developed. Once this was complete, two researchers collaboratively coded the findings. A third researcher randomly coded a sample of the documents in order to determine reliability.

We identified the dominant and biosecurity narratives that were used by the various actors who submitted evidence. We then compared the narratives, framing, and language used by the private sector with public and third sectors, and academia. We subsequently analysed the three main promoted 'remedies' to the AMR problem and categorised them within a 'market paradox' framework.

Discussion We found that, irrespective of sector, the submissions presented the problem of AMR similarly. The solutions, however, diverged dramatically. The relevant industries use particular discursive strategies to achieve their aims, including the development of market paradoxical positions; on the one hand, asking for subsidies and incentives, but on the other hand explaining that regulation would be detrimental to 'innovation'. We expand on these paradoxes, and catalogue the tactics used to achieve them discursively, including: obfuscating funding sources, stake inoculation, and lobbying for influence. Learnings from the unhealthy commodities industry allowed us to critically appraise the framing of industries involved in AMR.

Conclusion Overall, our CDA demonstrates that commercial interests deploying the crisis narratives do so in order to lobby heavily for self-serving solutions, namely deregulation and public subsidies. Discursive choices shaped by a technocratic-industry complex are redefining the pathways to success, monitoring, and decision-making in the global AMR arena.

\section{OP60 NATIONAL IMPLEMENTATION OF AN INTEGRATED DIABETES PROGRAMME IN IRELAND: REALIST EVALUATION}

${ }^{1} \mathrm{KN}$ O'Neill, ${ }^{1} \mathrm{FM}$ Riordan ${ }^{*},{ }^{1} \mathrm{E}$ Racine, ${ }^{2} \mathrm{ML}$ Tracey, ${ }^{3} \mathrm{C}$ Papoutsi, ${ }^{1} \mathrm{PM}$ Kearney, ${ }^{1}$ SM McHugh. 'School of Public Health, University College Cork, Cork, Ireland; ${ }^{2}$ Axis Consulting Limited, Hemel Hempstead, UK; ${ }^{3}$ Nuffield Department of Primary Care Health Sciences, University of Oxford, Oxford, UK

\subsection{6/jech-2020-SSMabstracts.59}

Background 'Integrated care' for chronic conditions is considered central to international health system reform. However, models of integrated care work differently in different circumstances. In Ireland, the National Diabetes Programme aimed to integrate diabetes care across primary, secondary and tertiary settings based on patient complexity through the introduction of new clinical posts and guidance for diabetes care. We conducted a realist evaluation to determine how and why the implementation of the programme worked (or not) across the country.

Methods Through documentary analysis and qualitative interviews $(n=19)$ with a purposive sample of national stakeholders, we developed an initial theory on how the programme was expected to work. We then refined this theory in semi-structured interviews $(n=39)$ with professionals purposively sampled to represent different clinical disciplines involved in implementation. We applied a realist logic of analysis and synthesis to iteratively build CMO configurations.

Results National stakeholders assumed that: 1) introducing guidance would formalise and standardise how care was provided, 2) that professionals would 'buy in' and align their work with new ways of working, and 3) that the new clinical posts would become catalysts for service changes at local level. At a national level, important contexts included varying levels of awareness about the programme, no plan for communicating service changes, and no established approach to implementation or professional oversight. Locally, experience delivering diabetes care, resource demands and familiarity with the intended purpose of the new clinical posts were important contextual factors. The extent to which integrated care was adopted and implemented depended on judgements made by health professionals (GPs, nurses, specialists and podiatrists) working in these contexts, specifically; judging the relative advantage of the programme and whether to engage in negotiations to legitimize their roles in diabetes care. 